\title{
LA FIN DE LA CROISADE DU PAPE CALIXTE III L'ASSASSINAT DU COMTE ULRICH DE CELLI ET LA FIN DE LA CROISADE DU PAPE CALIXTE III BORGIA (8 AVRIL 1456)
}

\author{
Por \\ GEORGES REYNAUD
}

La chute de Constantinople le 29 mai 1453 causa un grand effroi dans la chrétienté. Cependant, passés les premiers moments de stupeur et de consternation, cette cuisante blessure engendre un mouvement de riposte. Après une période d'atterrement, le pape Nicolas V proclama la guerre «juste», la guerre sainte, dans la bulle de croisade du 30 septembre 1453 uad perpetuam rei memoriam» (1). Le pape savait que pour que son appel ait un quelconque effet, la condition en était que règne la paix dans la péninsule italienne alors troublée par les rivalités entre républiques voire entre potentats locaux. La peur du Turc déclenchait à chaque événement un «réflexe» croisadiste dans les mentalités où l'idée du "passage d'oultremer» n'avait pas disparu. Mais l'efficacité nécessaire à une expédition demandait une unité absente pour I'heure du camp chrétien (2). Fort justement, Nicolas $V$ confia à un simple moine, Fra Simonetto da Camerino, la mission de sceller l'unité entre les principaux protagonistes italiens. Jusqu’à sa mort, le 24 mars 1455, il consacra ses efforts à réunir les conditions d'une expédition. L'aboutissement de son action se concrétise au traité de Lodi du 9 avril 1454, base de l'établissement d'une paix relativement durable en Italie et conséquence directe de la chute de Constantinople.

(1) Cf. Pastor, Hist. des Papes, Plon, Paris, 1888 T.2 p 254 - 255.

(2) A.S. Piccolomini Epistolae p. 127 l'évêque de Sienne écrit ainsi: "chaque état a son prince, chaque prince a ses intérêts particuliers. Quelle voix pourrait être assez éloquente pour réunir sous un même drapeau tant de puissances discordantes et hostiles\%. 
Dès lé 17 février, hors de la péninsule, on avait répondu à l'appel du Pape. Le .valeureux et puissant duc de Bourgogne s'était solennellement croisé lors du célèbre banquet du faisan devant les preux chevaliers de l'ordre de la Toison d'Or (3). Un grand mouvement était-il possible?

Parmi les grandes puissances de l'époque, Venise se déroba la première, signant un traité honteux avec les turcs le 18 avril 1454. Le roi de Naples, Alphonse d'Aragon, envoya quatre galères au Levant, qui n'y réalisèrent aucune action digne d'être rapportée. Le roi des Romains, l'empereur Frédéric III de Habsbourg, le glaive du "regnum" s'en tira en convoquant une diète à Ratisbonne. Le roi de France, Charles VII (4) répondit par la négative aux sollicitations de Zanon, I'évêque de Bayeux. Seul Philippe le Bon envoya le nouvel évêque d'Arras, Jean Jouffroy, auprès du roi de Naples pour préparer la croisade.

Un des rares événements favorables fut l'extension du traité de Lodi aux principales puissances italiennes le 2 mars 1455 (Venise, Milan, Florence, Naples et les états du Pape).

La diète de Ratisbonne se sépara après avoir décidé la convocation d'une nouvelle diète à Francfort pour la Saint Michel (29 septembre) où l'on espérait le présence de chefs expérimentés dans la lutte contre le Turc, deux hommes jouissant en ce domaine d'un grand prestige: le hongrois Jean Hunyadi et le ban de Croatie Ulrich de Cilli (5). Au cours des débats on parla d'une expédition énorme, de rassembler 200000 hommes. L'offensive devait être à la fois terrestre et maritime et personne n'avait contesté l'lutilité de cette action de guerre. Derrière la défense contre le turc resurgissait même l'idée de libérer les lieux saints (6).

L'union était en réalité quasi inexistąnte sur les détails de la réalisation d'un plan quil faisait I'unanimité quant à sơn but. Les dynasties étaient rivales et même ennemies; on renvoỹa le règlement de ces problèmes à une autre diète d'empire qui devait se tenir près de Vienne à Wiener-Neustadt. C'est pendant les assises de celle-ci que le pape mourut dans la nuit du 24 au 25 mars 1456. Près de trois ans avaient été perdus et aucune armée n'avait été avancée contre l'expansionnisme du jeune sultan Mahomet II.

Le désir de riposte de la chrétienté dans une guerre avant tout défensive contre les ottomans va se trouver ravivé par l'élection au siège de Saint Pierre du cardinal Alphonse Borgia.

(3) Cf. Olivier de la Marche, "Mémoires", publiées par la Société de l'Histoire de France H. Beaume et H d'Arbaumont Paris Renouard 1884 V.ll p.340 et aussi G. Cohen Scènes de la vie en France au Moyen Age Paris Lanore 1964 p. 45 - 60.

(4) Cf. Petit-Dutaillis: Charles VII Louis XI et la minorite de Charles VIII, Paris, Tallandier, 1981, p. $340-343$.

(5) Aujourd'hui Celje à la limite de la Slovénie et de la Croatie.

(6) Cf. Pastor op. cit p. 375 «Très Saint Père (écrivait Jean de Capistrano) le jour du salut s'est levé pour la chrétienté... non seulement nous aurons regagné l'empire grec et l'Europe, mais la Terre Sainte, Jérusalem seront aussi à nous.n 
Le premier représentant de cette célèbre famille est élu pape le 8 avril 1455 à l'âge de 77 ans et l'on donne peu de temps à vivre à ce vieillard malade. Pourtant, avant même d'avoir reçu les serments d'obédience, il montre une énergie farouche et se proclame ensuite le «rempart de la chrétienté». Jusqu'à sa mort, il va se consacrer à préparer la croisade. II y a d'ailleurs de quoi se préoccuper du turc: Rhodes, Trébizonde sont menacées; depuis l'été 1455 , les colonies de la Mer Noire sont tombées ou tributaires (Caffa). Seul Skanderberg semble pouvoir faire face en Albanie et reçoit à l'été 1455 I'aide de 1000 fantassins d'Alphonse d'Aragon.

Le 15 mai 1455 Calixte III fulmine la bulle "Ad summi apostolatus apicem" qui confirme les dispositions de la bulle du 30 septembre 1454 et supprime toutes les indulgences accordées depuis le Concile de Constance. Des légats partent dans toute la chrétienté. Alain de Coëtivy se rend auprès du roi de France; Juan de Carvajal en Allemange, Nicolas de Cues un prélat prestigieux doit se rendre en Angleterre. Des délégués spéciaux vont prêcher au petit peuple, frères mineurs ou moines mendiants tels Juan de Capistrano (7), Antoine de Montefalcone ou Robert de Lecce. Ces frères doivent en même temps récupérer les décimes et éviter les «indélicatesses" qui avaient eu lieu au temps des précédents pontifes; ces efforts ne furent pas vains puisque on évalue à 639000 ducats les sommes recouvrées pour la croisade; une flotte puissante put être construite de toutes pièces.

Le pape compte sur les plus farouches princes de l'occident. D'abord sur Philippe le Bon et pour le libérer, il confirme le 6 avril 1456 la paix francobourguignonne s'octroyant indirectement l'assentiment du roi de France, son suzerain nominal.

La situation sur le terrain empirait d'ailleurs. Le 14 juin 1455 Mahomet II s'était emparé de la riche ville minière serbe de Novo Brdo; tout le sud du despotat tomba ensuite. Le despote Georges Brancovic était alors à la diète de Raab (Györ) où l'on essayait d'organiser en Hongrie une riposte à l'ottoman. Continuant ses conquêtes, le sultan s'empara des salines d'Enos, de Thasos, de Samothrace et Imbros. Le 6 février 1456 à la diète de Buda, Juan de Carvajal essaya de redonner espoir en rapportant l'aide promise d'Alphonse $V$ de Naples et du duc Philippe de Bourgogne (8). Une indulgence fut accordée à tous ceux qui porteraient les armes contre le turc.

Le légat remit la croix à Capistrano ainsi qu'un bref papal pour prêcher la croisade. L'armée chrétienne devait partir le 6 avril. Les préparatifs trainè-

(7) Qui sera plus tard béatifié et connu sous le nom de Saint Jean de Capistran

(8) On a parfois contesté la profondeur des desseins croisadistes du Duc. Qu'il nous soit permis de rapporter l'opinion de G. Doutrepont admirable connaisseur de la Maison de Valois "En conséquence nous ne serons pas surpris si non loin de la tapisserie d'Hercule et de l'entremets de Jason, se dressent une église et Saint André avec sa croix. C'est précisément pour la même raison, soit donc parce que nous tenons compte de l'esprit du temps, que nous n'éprouvons aucune difficulté à penser que Philippe le Bon était plus qu'un croisé de théâtre ou de banquets. "Georges Doutrepont» "La littérature française à la cour des ducs de Bourgogne» Champion. Paris, 1909. 
rent et au début du mois de juillet le sultan avait investi les abords de Belgrade avant que les croisés n'y soient parvenus. Hunyadi et Capistrano ne purent atteindre la forteresse qu'en barque, mais leur arrivée redonna du moral aux assiégés, La bataille prit alors un tour singulier. Lorsque les janissaires attaquèrent le 21 juillet, Hunyadi, par une manoeuvre efficace, les laissa pénêtrer jusq'à la deuxième enceinte puis ses hommes regagnèrent le premier rempart et les assaillants coincés furent taillés en pièces. Le 22 à midi une sortie de quelques milliers de croisés derrière Juan de Capistrano provoqua l'effroi dans le camp ottoman qui fut abandonné; ce fut la déroute. Celle-ci ne fut malheureusement pas exploitée, la victoire ayant été si soudaine. II semble aussi que des désaccords soient apparus entre Hunyadi et le moine fanatique. La victoire fut ainsi sans lendemain du fait de la mort des principaux protagonistes: le 11 août 1456 Jean Hunyadi mourait vraisemblablement de la peste à Semlin sur le Danube (aujourd'hui Zemun, faubourg de Belgrade) et Capistrano lui survivait de peu jusqu'au 23 octobre où il mourut à llok. Connue dès le 9 août à Rome, la victoire de Belgrade connut un grand retentissement dans toute la chrétienté. Des "Te Deum» furent chantés dans toutes les églises de Rome dont les cloches sonnèrent à toute volée (9).

L'heure semblait venue pour Ulrich de Cilli, ennemi quasi constant de Hunyadi, de revenir au faîte de la gloire. Le ban de Croatie ne se méfiait guère des deux fils de Jean Hunyadi, Laszlo (ou Ladislas) (10) et Mathias. Le premier pourtant était demeuré à Belgrade tandis que son frère se trouvait dans l'arrière-pays. Cilli semble avoir essayé de s'entendre avec Ladislas dont la fiancée Elizabeth de Cilli, la propre fille d'Ulrich, était morte. Le comte Ulrich aurait proposé à Ladislas de l'adopter (11). Devant le refus du jeune homme, il se fit proclamer "lieutenant du roi pour la Hongrie» à la diète de Futak près de Peterwardein. La diète déclara aussi que seraient rendues au roi Ladislas le Posthume les places hongroises que les Hunyadi détenaient encore.

Ulrich de Cilli prit ainsi en compagnie du roi Ladislas la route de Belgrade pour se faire remettre la place où était resté le jeune Hunyadi et pour prendre le commandement des troupes chrétiennes. Le ban entrevovait peut-être alors une nouvelle victoire sur les turcs qui consacrerait son influence. Lui-même et le roi arrivèrent à Belgrade le 7 ou le 8 novembre 1456. IIs trouvèrent dit-on seulement deux cavaliers disant que Laszlo désirait qu'ils ne fussent accompagnés que d'une escorte peu nombreuse. Setton (12) affirme que le fils de Hunyadi aurait fait relever le pont levis avant que la totalité de l'escorte soit entrée. Le roi Ladislas le Posthume et le Comte furent admis dans une vaste salle vide ainsi que leurs gens; ils y mangèrent et dormirent. Laszlo vint leur

(9) En 1457 voulant perpétuer le souvenir de la bataille de Belgrade, Calixte Ill décréta que dorénavant le 6 août la fête de la Transfiguration de Notre Seigneur serait célébrée solennellement - Pastor op. cit p. 379

(10) Laszlo est la forme hongroise de Ladislas, Mathias deviendra plus tard roi de Hongrie sous le nom de Mathias Corvin

(11) F. Clement-Simon «Une grande famille en Europe centrale au XVe siècle: les comtes de Cilli». Revue d'histoire diplomatique janvier - mars 1930 p.21

112) K. Setton "The papacy and the Levant It" Philadephie, 1981, p. 183". 
apporter quelques paroles de bienvenue puss disparut. Cilli dormit avec sa cotte de mailles et les portes barricadées; elles furent parait-il secouées au milieu de la nuit. Le lendemain, le roi assista à la messe dans la chapelle de la forteresse. Vers la fin de l'office, Laslo fit dire à Ulrich de Cilli qu'il désirait lui parler. Ce dernier le suivit dans une pièce à l'écart. Laszlo s'en serait alors pris à lui violemment, l'accusant d'avoir traitreusement réuni la diète de Futak, d'être un étranger intrigant et imposteur. Ulrich aurait tiré son épée et blessé Laszlo. Ses h ommes de main postés autour de la pièce n'attendaient que cet instant et exécutèrent le Comte. Deux des amis d'Ulrich accoururent à ses cris mais ne purent intervenir; il fut lardé de coups (13). Les Hongrois coupèrent la tête du cadavre, la mirent dans un sac et l'envoyèrent aux turcs. Le sultan aurait répondu que partout on rendait les honneurs aux morts, que l'assaut serait donné si les restes de Cilli n'étaient pas traités avec respect. Laszlo se déchargea de la responsabilité de ces actes sur un subalterne et le cadavre complété fut enseveli avec solennité dans le domaine de Cilli. Laszlo laissa repartir le roi Ladislas qui l'assura qu'il ne le tiendrait pas responsable de cet assassinat.

Sur le coup, la nouvelle fut accueillie avec indignation dans l'armée du roi et parmi les croisés «et en un moment chacun eut revêtu son harnois et tous les capitaines se mirent à la tête de leurs gens et pensaient donner l'assaut au château» (14).

Le jeune roi Ladislas probablement fort affligé dut faire dire aux soldats qu'ils ne devaient pas intervenir dans l'affaire. L'armée campée entre la citadelle et la ville, exposée à être prise entre deux feux, par les turcs d'un côté et par les hongrois de l'autre, se trouvait là comme dans un cul de sac; au bout de quelques jours, le roi et le cardinal Carvajal donnèrent $d^{\prime}$ une commun accord aux croisés l'autorisation de rentrer en leurs pays. "Ainsi finit la campagne contre les Turcs par l'effet de la grande perfidie des seigneurs hongrois, que Dieu punisse!» (15).

Le jeune roi de Hongrie avait sans doute, lors des événements, fait contre mauvaise fortune bon coeur, mais il fut profondément touché de l'assassinat de son oncle. Dès le mois de mars 1457, saisissant le prétexte d'une conjuration, il fit arrêter à Buda Laszlo et Mathias Corvin et les traduisit en justice. L'aîné des Hunyadi fut condamné à mort. Mathias et ses amis à la prison. Ils y restèrent d'ailleurs jusqu'à la mort de Ladislas en novembre. Sur la mort de Laszlo, on rapporte un détail horrible: lors de son exécution, il aurait été encore vivant après avoir reçu trois coups du bourreau; il se serait alors relevé invoquant les lois qui ne permettent pas de donner plus de trois coups; c'est le roi lui-même qui aurait ordonné la continuation de l'exécution;

\footnotetext{
(13) Une description de l'exécution de Cilli est rapportée par Jacopo de Promontorio ce Campis in Babinger: Sitzungsaberichte d. Bayr. Akadem. du Wissen 1956 Heft b p 87 - 89 et aussi 1957 Heft 6 p 66 - 68

(14) Pastor, Hist. des Papes $T$ II, Plon, Paris, 1888, p 382

(15) Op. cit ibid. p. 383
} 
Les ennemis acharnés qu'étaient l'empereur Frédéric III de Hasbourg et le roi de Hongrie se déchirèrent alors pour obtenir les fiefs et domaine des Cilli puisque le Comte Ulrich n'avait pas d'héritiers. Sa veuve, Catherine Brancovic, désirait tout I'héritage mais le Habsbourg ne I'entendait pas de cette oreille. Il affirma que les biens de la famille comtale lui revenaient'en vertu" d'un contrat passé entre les deux maisons de Habsbourg et de Cilli stipulant que l'une devait recueillir la succession de l'autre, si l'une des deux venait à s'éteindre. L'empereur abandonna les biens provenant de la couronne de Hongrie et ne laissa à Catherine Brancovic que le seul château de Gurkfeld. La soeur de la sultane Mara n'y resta d'ailleurs pas et finit ses jours à Raguse.

Les démêlés successoraux de cette époque furent d'ailleurs un obstacle à l'exploitation militaire et politique de la bataille de Belgrade. On se disputa I'héritage de la famille de Cilli mais aussi celui de Georges Brancovic le vieux despote de Serbie. Ses trois fils, voire ses deux filles, s'opposèrent dans des conditions difficiles: les deux fils aînés, Grégoire et Etienne, avaient été rendus aveugles par les turcs; ce fut le troisième fils, Lazare, qui monta sur le trône de Serbie, mais il ne s'y maintint guère plus d'un an constamment acculé à s'opposer au nouveau gouverneur de Belgrade, Michel Szilagy, beaufrère de Jean Hunyadi. II mourut le 20 janvier 1458 sans héritier mâle. II était bien évidemment devenu impossible de poursuivre les Turcs.

La croisade du pape Calixte III Borgias'arrêtait là; une fois de plús, on. $n^{\prime}$ avait pu exploiter un net avantage militaire en raison d'incessantes rivalités internes (16). chées!

Que de possibilités la désunion des princes chrétiens avait-elle donc gâ-

(16) Calixte III essaya un temps de placer ce qui restait de la Serbie sous son autorié directe et donna à son légat le cardinal Carvajal des directives en ce sens. Setton Op cit p. 184 note 91. 